\title{
Stability of plane Poiseuille flow of viscoelastic fluids in the presence of a transverse magnetic field
}

\author{
Abdessamade RAFIKI, a and Ahmed HIFDI \\ Laboratory of Mechanics, University Hassan II-Casablanca, Morocco
}

\begin{abstract}
The linear stability of plan Poiseuille flow of an electrically conducting viscoelastic fluid in the presence of a transverse magnetic field is investigated numerically. The fourth-order Sommerfeld equation governing the stability analysis is solved by spectral method with expansions in lagrange's polynomials, based on collocation points of Gauss-Lobatto. The critical values of Reynolds number, wave number and wave speed are computed. The results are shown through the neutral curve. The main purpose of this work is to check the combined effect of magnetic field and fluid's elasticity on the stability of the plane Poiseuille flow. Based on the results obtained in this work, the magnetic field is predicted to have a stabilizing effect on the Poiseuille flow of viscoelastic fluids. Hence, it will be shown that for second-order fluids $(K<0)$, the critical Reynolds numbers $R e_{c}$ increase when the Hartman number $M$ increases for different values of elasticity number $K$, which is a known result. The more important result we have found, concerning second-grade fluids $(K>0)$ is that the critical Reynolds numbers $R e_{c}$ increase when the Hartman number $M$ increases for certain value of elasticity number $K$ and decrease for others. The latter result is in contrast to previous studies.
\end{abstract}

\section{Introduction}

The general nonlinear mechanical properties of the viscoelastic fluids greatly complicate the calculation of their hydrodynamic flow characteristics, specially when the flow is affected by various external factors such as a magnetic field serving as an effective mean of control. Some investigations had been made on the effect of a transverse magnetic field on the stability of plane Poiseuille flow. Lock [1] has simplified the governing equations of stability and has applied the method of asymptotic solutions and the result was that the magnetic field has a powerful stabilizing effect on the fluid flow. Following Lock, Takashima [2] has checked the validity of Lock's simplification and he has found that except the case when the magnetic Prandtl number $P_{m}$ is sufficiently small, the magnetic field has a stabilizing effect on the Newtonian fluid flow (at least in the range of the Hartman numbers $M$ covered in this work). The role played by the elasticity of viscoelastic fluids in the transition from a laminar to turbulent flow has been investigated by Shun and Schwarz [3], then by Sadeghy et al.[4]. It is concluded that for plan Poiseuille flow of second grade fluids $(K>0)$ is identically stable and this flow of second order fluids $(K<0)$ is instable. This problem has been treated in the presence of a magnetic field by Carlsson and Lundgren [5]. They have shown some results of the stability of second grade Rivlin-Ericksen magnetofluid flow, between parallel planes in the cases when the imposed magnetic field is either parallel to the flow direction and perpendicular to the planes. It has been seen that the magnetic field has a stabilizing effect on the flow in the case of a coplanar magnetic field. However, they did not give any result about the transverse magnetic field.

In the present work, we reexamine in a greater detail and

a e-mail: , abs.rafiki@gmail.com we extend the problem first treated by Carlsson and Lundgren [5]; when the magnetic field is perpendicular to the planes. Our study mainly focuses on the combined effect of magnetic field and fluid's elasticity on the Poiseuille flow. A simplification of the stability equations, that has been made by Lock [1] is used in our problem; considering the fact that the magnetic Prandtl number $P_{m}$ for most electrically conducting fluids is extremely small. The spectral method based on collocation points of Gauss-Lobatto [canuto et al.] [6] is used to solve the resulting stability equations. Besides, to make the work more tangible, we examine the behavior of the most dangerous mode for different values of $M$ and $K$. The stability of the flow is defined as the sign of the imaginary part of the eigenmodes and its large absolute value. These investigations show that the transversal magnetic field has a stabilizing effect on the Poiseuille flow of the second order fluids $(K<0)$. In contrast, the magnetic field has both stabilizing and destabilizing effects on the Poiseuille flow of second-grade fluids $(K>0)$.

\section{Governing equations and dimensionless parameters}

We consider a plane Poiseuille flow of an electrically conducting fluid between two parallel plates in the presence of a uniform external magnetic induction $B_{0}$ perpendicular to the plates for a second-grade and second-order models. A Cartesian co-ordinate system $\left(x^{*}, y^{*}, z^{*}\right)$ is chosen such that the origin, 0 , is a midway between the plates with the $\mathrm{x}$-axis in the direction of the flow, the $\mathrm{y}$-axis perpendicular to the plates, and $z$ the neutral direction. the plates are assumed at $y= \pm h$, and the uniform magnetic induction $B$ has the components $\left(0, B_{0}, 0\right)$. 
The equations governing fluid motion in dimensionless form are given by:

$$
\begin{gathered}
{\left[\frac{\partial V}{\partial t}+V \cdot \nabla \mathbf{V}\right]=-\nabla P+\nabla \cdot \tau+H a \nabla \times(B \times B)} \\
\frac{\partial B}{\partial t}=\nabla \times(V \times B)+\frac{1}{R e P m}\left(\nabla^{2} B\right) \\
\nabla . V=0 \quad \nabla \cdot B=0
\end{gathered}
$$

The extra stress $\tau$ is defined by:

$$
\tau=\frac{1}{R e} A_{1}+K_{1} A_{2}+K_{2} A_{1}^{2}
$$

We used here the following dimensionless variables after dropping the sign $*$ over the variables:

$$
\begin{gathered}
\left(x^{*}, y^{*}, z^{*}\right)=\left(\frac{x}{h}, \frac{y}{h}, \frac{z}{h}\right) ; t^{*}=\frac{U_{0} t}{h} ; \quad V^{*}\left(u^{*}, v^{*}\right)=V\left(\frac{u}{U_{0}}, \frac{v}{U_{0}}\right) \\
B^{*}=\frac{B}{B_{0}} ; \quad P^{*}=\frac{P}{\rho U_{0}^{2}} ; \tau^{*}=\frac{\tau}{\rho U_{0}^{2}}
\end{gathered}
$$

In the above equations, the Reynolds number, the elasticity number, the magnetic pressure number, and the Prandtl number are defined respectively as:

$R e=\frac{h U_{0}}{v} ; \quad K_{i}=\frac{\alpha_{i}}{\rho h^{2}}: i=1,2 ; \quad H a=\frac{B 0^{2}}{\mu_{0} \rho U_{0}^{2}} ; \quad P_{m}=\mu_{0} \sigma v$

where $\rho$ is the fluid density, $v$ is the kinematic viscosity, $U_{0}$ is the velocity at the center, $\alpha_{i}$ is the normal stress module time, $\sigma$ is the electrical conductivity, $\mu_{0}$ is the magnetic permeability and $A_{i}$ is kinematical tensors.

The no-slip condition is satisfied by the velocity at the plates, while for the magnetic field, the plates are assumed to be electrically non-conducting. Since we shall restrict our study of the stability analysis to two-dimensional perturbations, the solution for fully developed Poiseuille flow between two plates in two-dimensional flow $(x, y)$ is:

$$
\begin{gathered}
U_{b}=\frac{\cosh (M)-\cosh (M y)}{\cosh (M)-1} \\
B_{x b}=\frac{R_{m}}{M(\cosh (M)-1)}[\sinh (M y)-y \sinh (M)]
\end{gathered}
$$

where $M$ is the non-dimensional quantity $B_{0} h \sqrt{\sigma / \rho v}$ which call the Hartmann number, and $R_{m}=R e P_{m}=U_{0} h \mu_{0} \sigma$ is the magnetic Reynolds number.

\section{Stability analysis}

\subsection{Linear stability}

we now perturb the steady-state solution and perform the standard normal mode analysis to look for the solution in the form:

$U=U_{b}+u \quad V=v \quad P=P_{b}+p \quad B_{x}=B_{x_{b}}+b_{x} \quad B_{y}=B_{0}+b_{y}$
Having introduced a perturbation stream function $\psi$, and decomposed all perturbations into its Fourier modes as:

$$
\left[\psi, b_{x}, b_{y}\right]=[\phi, \delta, \theta] e^{i \alpha(x-c t)}
$$

where $\phi, \delta, \theta$ are the (complex) amplitudes of the perturbations $\psi, b_{x}, b_{y}$ respectively, $\alpha$ is the wave number and $c$ is the complex wave speed.

Applying the assumptions (7) and (8) in the Eq.(1) and the $y$ component of Eq.(2), neglecting second and higher order of the perturbation, we can find the following equations:

$$
\begin{gathered}
{\left[1+i \alpha \operatorname{KRe}\left(U_{b}-c\right)\right]\left(D^{2}-\alpha^{2}\right)^{2} \phi-i \alpha \operatorname{Re}\left[\left(U_{b}-c\right)\left(D^{2}-\alpha^{2}\right)\right.} \\
\left.-\left(D^{2} U_{b}-K D^{4} U_{b}\right)\right] \phi=H a \operatorname{Re}\left[-\frac{D}{i \alpha}\left(D^{2}-\alpha^{2}\right)+D^{2} B_{x_{b}}\right. \\
\left.-B_{x_{b}}\left(D^{2}-\alpha^{2}\right)\right] \theta \\
i \alpha\left(U_{b}-c\right) \theta-\alpha^{2} B_{x_{b}} \phi+i \alpha D \phi=\frac{1}{R_{m}}\left(D^{2}-\alpha^{2}\right) \theta
\end{gathered}
$$

Above $D$ represents differentiation with respect to the wallnormal coordinate $(y)$.

According to Lock [1] for most electrically conducting fluids, the magnetic Reynolds number $R_{m}$ is usually very small, and thanks to this assumption, all terms contain $B_{x_{b}}$ will vanish in Eq.(9) and Eq.(10) and also the term $\left(U_{b}-c\right) \theta$ in Eq.(10). Thus, the stability equation can be written in the following form as:

$$
A \phi=c B \phi \quad \phi( \pm 1)=D \phi( \pm 1)
$$

Where

$$
\begin{gathered}
A=\left(1+i \alpha K \operatorname{Re} U_{b}\right)\left(D^{2}-\alpha^{2}\right)^{2}-i \alpha \operatorname{Re}\left[U_{b}\left(D^{2}-\alpha^{2}\right)\right. \\
\left.-\left(D^{2} U_{b}-K D^{4} U_{b}\right)\right]+M^{2} D^{2}
\end{gathered}
$$

and $\quad B=i \alpha K \cdot \operatorname{Re}\left(D^{2}-\alpha^{2}\right)^{2}-i \alpha \operatorname{Re}\left(D^{2}-\alpha^{2}\right)$

for $M=0$ and $K=0$, Eq.(11) is in the form of OrrSommerfeld equation of ordinary hydrodynamics.

\section{2 numerical solutions}

For specified values of $M, K, \operatorname{Re}$, and $\alpha$. Eq.(11) define an eigenvalue problem for $c$. To solve it, we adopt the Tchebychev spectral method based on the most commonly used collocation points of Gauss-Lobatto. For a complete description of this method, we refer to Canuto et al.[6] To validate our code of calculation, our results agree with those obtained by Takashima [2] using the Chebyshev collocation method for Newtonian fluid under a transverse magnetic field. The code is also tested again with the results obtained by Sadeghy et al.[4] when they used the collocation method for the Second-order fluids $(K<0)$, and the Riccati method in the case of second-grade fluids $(K>0)$. Their results are in good agrement with ours presented in Tables 1 and 2 when $M=0$.

\section{Results and discussion}

Having validated our code, we are now at the stage of presenting our instability results depicting the effects of fluid elasticity and the magnetic field on the stability of plan Poiseuille flow. In the following two subsections, we present the results of the second-order $(K<0)$ and the second-grade $(K>0)$ fluids. 
Table 1. Variation of critical values of $\alpha$, and $R e$ versus $M$ at different values of $K$ for the second-order fluid.

\begin{tabular}{lllll}
\hline & $K=-10^{-5}$ & \multicolumn{3}{l}{$K=-5.10^{-5}$} \\
\hline $\mathrm{M}$ & $\operatorname{Re}_{c}$ & $\alpha_{c}$ & $R e_{c}$ & $\alpha_{c}$ \\
\hline 0 & 5637.5443 & 1.027 & 5166.9226 & 1.05 \\
1 & 9450.0482 & 0.98 & 8285.3775 & 1.016 \\
2 & 25235.6321 & 0.95 & 19372.3961 & 1.02 \\
3 & 52574.9773 & 1.01 & 33803.5376 & 1.14 \\
4 & 80814.0654 & 1.13 & 41812.1753 & 1.45 \\
\hline & $K=-10^{-4}$ & & $K=-2.10^{-4}$ & \\
\hline $\mathrm{M}$ & $R e_{c}$ & $\alpha_{c}$ & $R e_{c}$ & $\alpha_{c}$ \\
\hline 0 & 4697.8042 & 1.08 & 4014.1200 & 1.13 \\
1 & 7234.0080 & 1.05 & 5860.2324 & 1.11 \\
2 & 15243.2114 & 1.09 & 11119.4471 & 1.19 \\
3 & 24057.2829 & 1.27 & 16679.0148 & 1.47 \\
4 & 29338.3993 & 1.66 & 21280.0522 & 2.00 \\
\hline
\end{tabular}

\subsection{Second-order fluid}

It is seen from Figure 1 and Table 1 that at fixed Hartmann number $M$, the minimum critical Reynolds number $R e_{c}$ decreases and the critical wave number $\alpha_{c}$ increase by an increase in the absolute value of elasticity number $K$. While at fixed $K$ and by an increase $M$, the $\operatorname{Re}_{c}$ and $\alpha_{c}$ are also increased. This highlights that the existence of the elasticity in the fluids has a destabilizing effect on the second-order fluid flow, which is stabilized by an imposed transverse magnetic field.

Figure 4 depicts the effects of magnetic field and elasticity number $K$ on the most dangerous eigenmodes at $R e=$ 10000 and $\alpha=1$. This figure shows that the magnitude of imaginary part of the eigenvalue $\left(\left|c_{i}\right|\right)$ increases gradually with the increase of the absolute value of elasticity number $K$ and decreases when the Hartmann number $M$ increases. Hence, we conclude that the fluid elasticity has a destabilizing effect and the transversal magnetic field has a stabilizing effect on this eigenmode. The eigenmode is highly unstable at this range of Reynolds number and wave number, as noted by the large value of imaginary part $c_{i}$ and its positive sign.

\subsection{Second-grade fluid}

Figure 2 shows the effect of magnetic field (Hartmann number $M$ ) on the neutral stability curves at different values of elasticity number $K$. Also, Table 2 presents results depicting the effects of magnetic field and fluids elasticity on the critical threshold. As can be seen in Figure 2 and Table 2. At fixed $M$, the critical Reynolds number $R e_{c}$ is increased by an increase in the elasticity number $K$. Hence, the second-grade fluids elasticity has a stabilizing effect on the Poiseuille flow. For $K=10^{-5}$, we notice from Figure 3 that the magnetic field has a stabilizing effect on the second-grade fluids flow. The most important results we have found (see Table 2, when $K=5.10^{-5}$ and $10^{-4}$ ) are that in the range of $M<3$ the critical Reynolds number $R e_{c}$ is increased by an increase in the Hartmann number $M$. However, in the range of $3<M<4$ the critical Reynolds number $R e_{c}$ is decreased by an increase in
Table 2. Variation of critical values of $\alpha$, and $\operatorname{Re}$ versus $M$ at different values of $K$ for the second-grade fluid.

\begin{tabular}{lllll}
\hline \multicolumn{3}{l}{$K=10^{-5}$} & \multicolumn{3}{l}{$K=5.10^{-5}$} \\
\hline $\mathrm{M}$ & $R e_{c}$ & $\alpha_{c}$ & $R e_{c}$ & $\alpha_{c}$ \\
\hline 0 & 5915.1659 & 1.0143 & 6586.7092 & 0.9875 \\
1 & 10193.3896 & 0.9651 & 12220.6476 & 0.9245 \\
2 & 30227.8124 & 0.9110 & 55467.2233 & 0.7965 \\
3 & 77598.3051 & 0.9126 & 4499738.6773 & 0.3706 \\
4 & 167390.6290 & 0.9314 & 3788705.3556 & 0.4453 \\
\hline & $K=10^{-4}$ & & $K=2.10^{-4}$ & \\
\hline $\mathrm{M}$ & $R e_{c}$ & $\alpha_{c}$ & $R e_{c}$ & $\alpha_{c}$ \\
\hline 0 & 7755.5900 & 0.9494 & 13389.9695 & 0.8412 \\
1 & 1725184.4174 & 0.3667 & 2397556.2385 & 0.3051 \\
2 & 2933446.8750 & 0.4378 & 3788264.7986 & 0.3554 \\
3 & 6343946.5278 & 0.2689 & 103060537.9942 & 0.0420 \\
4 & 5691207.4500 & 0.3152 & & \\
\hline
\end{tabular}

Hartmann number $M$. Hence, we conclude that the magnetic field has a stabilizing and destabilizing effect on the second-grade fluids flow. When $K=2.10^{-4}$, the critical Reynolds number $R e_{c}$ increases gradually with increases of the Hartmann number $M$. Therefore, the second-grade fluids flow becomes extremely stable.

At $R e=10000$ and $\alpha=1$, Figure 4 shows that the magnitude of imaginary part of the most dangerous eigenmode $\left(\left|c_{i}\right|\right)$ decreases with the increase of Hartmann number $M$ up to a certain values of $M=3$ and then it increases with the increase of $M$ at different values of elasticity number $K$. Therefore, the magnetic field has a stabilizing and destabilizing effect on this eigenmode. Also, we see from this figure, when $M<3$, the magnitude of imaginary part of this eigenmode decreases with the elasticity number $K$ increases. Hence, the fluid's elasticity has a stabilizing effect on this eigenmode. Interestingly, as shown in figure 4, an increase or decrease in the elasticity number appears to have no major effect on the magnitude of $c_{i}$ for $3<M<4$. The most dangerous eigenmode is stable in the case when $K=10^{-4}$ and $2.10^{-4}$ as noted by the negative value of $c_{i}$ at various values of $M$, and in the case when $K=10^{-5}$ and $5.10^{-5}$ the most dangerous eigenmode is unstable respectively for $M<1$ and $M<0.7$, and then it becomes stable below these values of $M$.

\section{Conclusion}

A linear stability analysis of plane Poiseuille flow of both second-order $(K<0)$ and second-grade $(K>0)$ fluids has been presented. The critical numerical values of Reynolds number $R_{c}$, wave number $\alpha_{c}$ and wave speed $c=c_{r}+i c_{i}$ were obtained for several selected values of the Hartmann number and elasticity number.

For the second-order fluid flow, we can conclude that the magnetic field has a stabilizing effect on the flow and this effect decreases as elasticity number increases. Besides, for the most dangerous eigenmode at $R e=10000$ and $\alpha=1$, it has been found that as the elasticity number $K$ increases: that is as we approach towards Newtonian fluid limit, the most dangerous eigenmode became more stable, 
however no stabilization does not occurs, even by increase in the Hartmann number $M$.

The results presented in this work for second-grade fluid flow, reveal in the first place that the fluids elasticity has a stabilizing effect on the Poiseuille flow. Moreover, the magnetic field has been predicted to have the same effect on this flow. However, a change in the Hartmann number $M$ leads to a considerable change of the critical Reynolds number $R e_{c}$. Consequently, a sudden destabilization of this flow can be observed at certain values of elasticity number $\left(5.10^{-5}<K<10^{-4}\right)$. We can conclude that the combined effect of second-grade fluid elasticity and the transverse magnetic field is to stabilize or destabilize the plane Poiseuille flow. Secondly, at $\operatorname{Re}=10000$ and $\alpha=1$, the fluids elasticity has a stabilizing effect on the most dangerous eigenmode for various values of Hartmann number lower than $M=3$, and then in the range of $3<M<4$, the magnetic field has a destabilizing effect on this eigenmode. However, the fluid elasticity has no effect in this range of Hartmann number $M$.

Finally, it can be noted that our conclusion are agree with the previous known results saying that the transverse magnetic field has a stabilizing effect on the plane Poiseuille flow (Lock [1], Takashima [2], Carlson et al. [5]), and the elasticity of second-order fluids has an opposed effect (Shun et al.[3], Sadeghy et al.[4]), and that of second-grade fluids has a stabilizing effect in this flow (Sadeghy et al.[4]). In addition, we are the first to study the combined effect of the magnetic field and fluid's elasticity on the stability of Poiseuille flow of second-order and second-grade fluids, and depicting this effect on the most dangerous eigenmode of fundamental mode defined as $\operatorname{Re}=10000$ and $\alpha=1$.

\section{References}

1. Lock R.C., The stability of the flow of an electrically conducting fluid between parallel planes under a trasverse magnetic field, Proc. Roy. Soc. Lond. A 221, (1955) 105-125.

2. Takashima M., The stability of modified plane Poiseuille flow in the presence of a transverse magnetic field, Fluid Dyn. Res. 17, (1996) 293-310.

3. Chun D.H. and Schwarz W.H., Stability of plane Poiseuille flow of a second-order fluid, Phys. Fluids 11, (1968) 5-9.

4. Sadeghy K., Taghavi S.M., Khabazi N., Mirzadeh M.and Karimfazli I., On the use of hydrodynamic instability test as an efficient tool for evaluating viscoelastic fluid models, ASME J. Basic. Engng. 1, (2007) 367-379.

5. Carlsson A., Lundgren E., Stability of second grade Rivlin-Ericksen magnetofluid flow between parallel planes in presence of a magnetic field, Physica Scripta 7, (1973) 177-178.

6. Canuto C., Hussaini M.Y., Quarteroni A. and ZANG T.A., Spectral Methods in Fluid Dynamics (SpringerVerlag,1988)
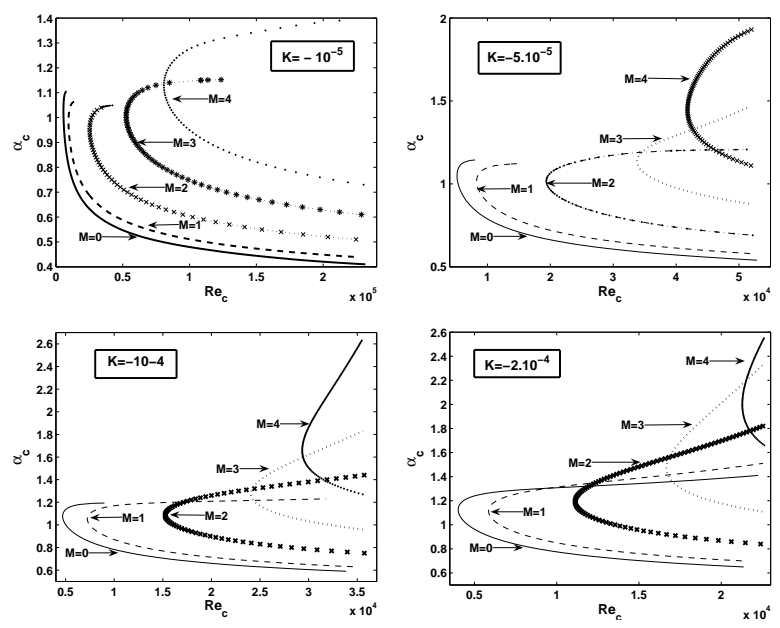

Fig. 1. Neutral stability curve for various values of Hartmann number $M$ at different values of elasticity number $K$ of the second-order fluid flow
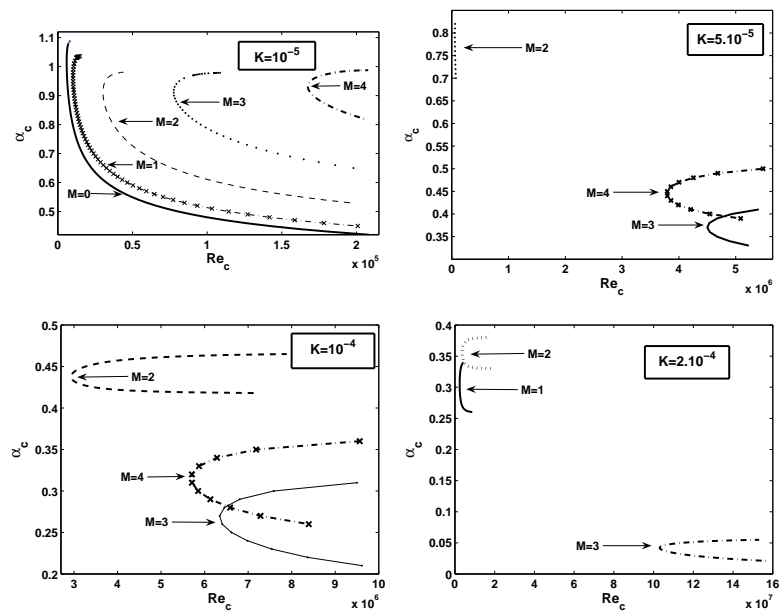

Fig. 2. Neutral stability curve for various values of Hartmann number $M$ at different values of elasticity number $K$ of the second-grade fluid flow
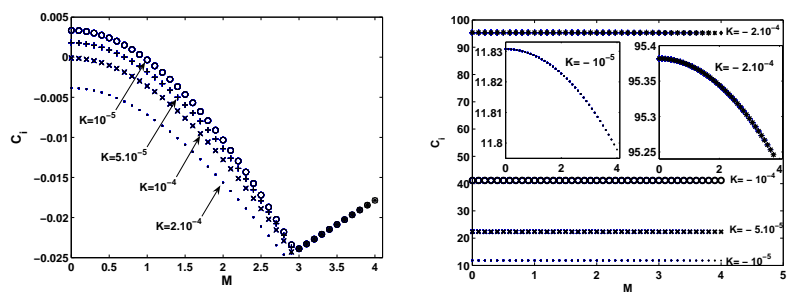

Fig. 3. The effect of the Hart- Fig. 4. The effect of the Hartmann number on the imagi- mann number on the imaginary part of the most dangerous nary part of the most dangerous eigenmode at $R e=10000$ and eigenmode at $R e=10000$ and $\alpha=1$ for the second grade fluid $\alpha=1$ for the second order fluid 